\title{
FUZZY SUPER IRRESOLUTE FUNCTIONS
}

\author{
S. E. ABBAS \\ Received 26 December 2002
}

\begin{abstract}
The concept of fuzzy super irresolute function was considered and studied by Šostak's (1985). A comparison between this type and other existing ones is established. Several characterizations, properties, and their effect on some fuzzy topological spaces are studied. Also, a new class of fuzzy topological spaces under the terminology fuzzy $S^{*}$-closed spaces is introduced and investigated.
\end{abstract}

2000 Mathematics Subject Classification: 54A40.

1. Introduction and preliminaries. Šostak [10], introduced the fundamental concept of a fuzzy topological structure, as an extension of both crisp topology and Chang fuzzy topology [1], in the sense that not only the objects are fuzzified, but also the axiomatics. In [11, 12], Šostak gave some rules and showed how such an extension can be realized. Chattopadhyay et al. [2, 3] have redefined the same concept. In [8], Ramadan gave a similar definition, namely "smooth topological space." It has been developed in many directions $[4,5,6,7,13]$.

In the present note, some counterexamples and characterizations of fuzzy super irresolute functions are examined. It is seen that fuzzy super irresolute function implies each of fuzzy irresolute [9] and fuzzy continuity [10], but not conversely. Also, properties preserved by fuzzy super irresolute functions are examined. Finally, we define a fuzzy $S^{*}$-closed space in fuzzy topological spaces in Šostak sense and characterize such a space from different angles. Our aim is to compare the introduced type of fuzzy covering property with the existing ones.

Throughout this note, let $X$ be a nonempty set, $I=[0,1]$, and $I_{\circ}=(0,1]$. For $\alpha \in I, \underline{\alpha}(x)=\alpha$ for all $x \in X$. The following definition and results which will be needed.

DEFinition 1.1 [10]. A function $\tau: I^{X} \rightarrow I$ is called a fuzzy topology on $X$ if it satisfies the following conditions:

(1) $\tau(\underline{0})=\tau(\underline{1})=1$,

(2) $\tau\left(\mu_{1} \wedge \mu_{2}\right) \geq \tau\left(\mu_{1}\right) \wedge \tau\left(\mu_{2}\right)$ for any $\mu_{1}, \mu_{2} \in I^{X}$,

(3) $\tau\left(\bigvee_{i \in \Gamma} \mu_{i}\right) \geq \bigwedge_{i \in \Gamma} \tau\left(\mu_{i}\right)$ for any $\{\mu\}_{i \in \Gamma} \subset I^{X}$.

The pair $(X, \tau)$ is called a fuzzy topological space (FTS). 
REMARK 1.2. Let $(X, \tau)$ be an FTS. Then, for each $\alpha \in I, \tau_{\alpha}=\left\{\mu \in I^{X}\right.$ : $\tau(\mu) \geq r$ is a Chang's fuzzy topology on $X$.

THEOREM 1.3 [3]. Let $(X, \tau)$ be an FTS. Then, for each $r \in I_{\circ}$ and $\lambda \in I^{X}$, an operator $C_{T}: I^{X} \times I_{\circ} \rightarrow I^{X}$ is defined as follows:

$$
C_{\tau}(\lambda, r)=\bigwedge\left\{\mu \in I^{X}: \lambda \leq \mu, \tau(\underline{1}-\mu) \geq r\right\} .
$$

For $\lambda, \mu \in I^{X}$ and $r, s \in I_{\circ}$, the operator $C_{T}$ satisfies the following conditions:

(1) $C_{T}(\underline{0}, r)=\underline{0}, \lambda \leq C_{T}(\lambda, r)$,

(2) $C_{T}(\lambda, r) \vee C_{T}(\mu, r)=C_{T}(\lambda \vee \mu, r)$,

(3) $C_{T}(\lambda, r) \leq C_{T}(\lambda, s)$ if $r \leq s$,

(4) $C_{T}\left(C_{T}(\lambda, r), r\right)=C_{T}(\lambda, r)$.

THEOREM 1.4 [9]. Let $(X, \tau)$ be an FTS. Then, for each $r \in I_{\circ}$ and $\lambda \in I^{X}$, an operator $I_{\tau}: I^{X} \times I_{\circ} \rightarrow I^{X}$ is defined as follows:

$$
I_{\tau}(\lambda, r)=\bigvee\left\{\mu \in I^{X}: \lambda \geq \mu, \tau(\mu) \geq r\right\}
$$

For $\lambda, \mu \in I^{X}$ and $r, s \in I_{\circ}$, the operator $I_{\tau}$ satisfies the following conditions:

(1) $I_{\tau}(\underline{1}-\lambda, r)=\underline{1}-C_{\tau}(\lambda, r)$,

(2) $I_{\tau}(\underline{1}, r)=\underline{1}, \lambda \geq I_{\tau}(\lambda, r)$,

(3) $I_{\tau}(\lambda, r) \wedge I_{\tau}(\mu, r)=I_{\tau}(\lambda \wedge \mu, r)$,

(4) $I_{\tau}(\lambda, r) \geq I_{\tau}(\lambda, s)$ if $r \leq s$,

(5) $I_{\tau}\left(I_{\tau}(\lambda, r), r\right)=I_{\tau}(\lambda, r)$.

DEFINITION 1.5 [9]. Let $(X, \tau)$ be an FTS. Then, for each $r \in I_{\circ}$ and $\lambda \in I^{X}$, the following statements hold:

(1) $\lambda$ is called $r$-fuzzy semi-open ( $r$-FSO) if there exists $v \in I^{X}$ with $\tau(v) \geq r$ such that $v \leq \lambda \leq C_{\tau}(\nu, r)$; equivalently, $\lambda \leq C_{T}\left(I_{\tau}(\lambda, r), r\right)$;

(2) $\lambda$ is called $r$-fuzzy semiclosed ( $r$-FSC) if there exists $v \in I^{X}$ with $\tau$ ( $1-$ $\nu) \geq r$ such that $I_{\tau}(\nu, r) \leq \lambda \leq \nu$; equivalently, $I_{\tau}\left(C_{\tau}(\lambda, r), r\right) \leq \lambda$;

(3) $\lambda$ is called $r$-fuzzy semiclopen ( $r$-FSCO) if $\lambda$ is $r$-FSO and $r$-FSC;

(4) $\lambda$ is called $r$-fuzzy regular open $\left(r\right.$-FRO) if $\lambda=I_{\tau}\left(C_{\tau}(\lambda, r), r\right)$;

(5) the $r$-fuzzy semi-interior of $\lambda$, denoted $\operatorname{SI}_{\tau}(\lambda, r)$, is defined by $\operatorname{SI}_{\tau}(\lambda, r)=$ $\bigvee\left\{v \in I^{X}: v \leq \lambda, v\right.$ is $r$-FSO $\}$

(6) the $r$-fuzzy semiclosure of $\lambda$, denoted $\mathrm{SC}_{\tau}(\lambda, r)$, is defined by $\mathrm{SC}_{\tau}(\lambda, r)=$ $\bigwedge\left\{v \in I^{X}: v \geq \lambda, v\right.$ is $r$-FSC $\}$.

THEOREM 1.6 [9]. Let $(X, \tau)$ be an FTS. For $\lambda \in I^{X}$ and $r \in I_{\circ}$, the following statements are valid:

(1) $\lambda$ is $r$-FSO if and only if $\lambda=\operatorname{SI}_{\tau}(\lambda, r)$, and $\lambda$ is $r$-FSC if and only if $\lambda=$ $\mathrm{SC}_{T}(\lambda, r)$;

(2) $I_{\tau}(\lambda, r) \leq \mathrm{SI}_{\tau}(\lambda, r) \leq \lambda \leq \mathrm{SC}_{\tau}(\lambda, r) \leq C_{\tau}(\lambda, r)$; 
(3) $\mathrm{SC}_{T}\left(\mathrm{SC}_{T}(\lambda, r), r\right)=\mathrm{SC}_{T}(\lambda, r)$;

(4) $C_{T}\left(\mathrm{SC}_{T}(\lambda, r), r\right)=\mathrm{SC}_{T}\left(C_{T}(\lambda, r), r\right)=C_{T}(\lambda, r)$;

(5) $\mathrm{SI}_{\tau}(\underline{1}-\lambda, r)=\underline{1}-\mathrm{SC}_{\tau}(\lambda, r)$.

LEMMA 1.7. For any fuzzy set $\lambda$ in an $\operatorname{FTS}(X, \tau)$ and $r \in I_{\circ}$, if $\tau(\lambda) \geq r$, then $I_{\tau}\left(C_{T}(\lambda, r), r\right)=\mathrm{SC}_{\tau}(\lambda, r)$.

Proof. Since $\mathrm{SC}_{T}(\lambda, r)$ is $r$-FSC, $I_{\tau}\left(C_{\tau}\left(\mathrm{SC}_{\tau}(\lambda, r), r\right), r\right) \leq \mathrm{SC}_{\tau}(\lambda, r)$ and hence, by Theorem 1.6(4), $I_{\tau}\left(C_{T}(\lambda, r), r\right) \leq \mathrm{SC}_{\tau}(\lambda, r)$. To prove the opposite inclusion, since $\tau(\lambda) \geq r, r \in I_{\circ}$, we have $\lambda \leq I_{T}\left(C_{T}(\lambda, r), r\right)$ so that $1-\lambda \geq$ $\underline{1}-I_{\tau}\left(C_{T}(\lambda, r), r\right)=C_{T}\left(I_{\tau}(\underline{1}-\lambda, r), r\right)$. But $C_{T}\left(I_{T}(\underline{1}-\lambda, r), r\right)$ is $r$-FSO. Hence $C_{\tau}\left(I_{\tau}(\underline{1}-\lambda, r), r\right) \leq \mathrm{SI}_{\tau}(\underline{1}-\lambda, r)$ and $\operatorname{so~SC}_{\tau}(\lambda, r) \leq I_{\tau}\left(C_{\tau}(\lambda, r), r\right)$.

DEFINITION 1.8. Let $(X, \tau)$ and $(Y, \eta)$ be FTSs and let $f: X \rightarrow Y$ be a function which is called

(1) fuzzy continuous (FC) if and only if $\eta(\mu) \leq \tau\left(f^{-1}(\mu)\right)$ for each $\mu \in I^{Y}$ [10],

(2) fuzzy open if and only if $\tau(\lambda) \leq \eta(f(\lambda))$ for each $\lambda \in I^{X}$ [10],

(3) fuzzy semicontinuous (FSC) if and only if $f^{-1}(\mu)$ is $r$-FSO set of $X$ for each $\eta(\mu) \geq r, r \in I_{\circ}$ [9],

(4) fuzzy irresolute (FI) if and only if $f^{-1}(\mu)$ is $r$-FSO set of $X$ for each $\mu$ is $r$-FSO set of $Y, r \in I$ 。 [9].

\section{Fuzzy super irresolute functions}

DeFinITION 2.1. Let $(X, \tau)$ and $(Y, \eta)$ be FTSs and let $f: X \rightarrow Y$ be a function which is called

(1) fuzzy super irresolute (F-super I) if and only if $\tau\left(f^{-1}(\mu)\right) \geq r$ for each $\mu$ is $r$-FSO set of $Y, r \in I_{\circ}$,

(2) fuzzy completely continuous (FCC) if and only if $f^{-1}(\mu)$ is $r$-FRO set of $X$ for each $\mu \in I^{Y}$ and $\eta(\mu) \geq r, r \in I_{\circ}$,

(3) fuzzy completely irresolute (FCI) if and only if $f^{-1}(\mu)$ is $r$-FRO set of $X$ for each $r$-FSO set $\mu \in I^{Y}$ and $r \in I_{\circ}$.

REMARK 2.2. One can show the connection between these types and other existing ones by the following diagram:

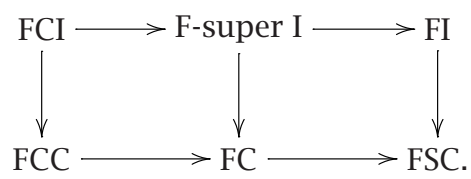

The converse of the previous implications need not be true in general as shown in the following counterexample. 
CounterexAmple 2.3. Let $\mu_{1}, \mu_{2}$, and $\mu_{3}$ be fuzzy subsets of $X=\{a, b, c\}$ defined as follows:

$$
\begin{array}{lll}
\mu_{1}(a)=0.9, & \mu_{1}(b)=0.0, & \mu_{1}(c)=0.1, \\
\mu_{2}(a)=0.9, & \mu_{2}(b)=0.7, & \mu_{2}(c)=0.2, \\
\mu_{3}(a)=0.9, & \mu_{3}(b)=0.3, & \mu_{3}(c)=0.2 .
\end{array}
$$

Then $\tau, \eta: I^{X} \rightarrow I$, defined as

$$
\tau(\lambda)=\left\{\begin{array}{ll}
1, & \text { if } \lambda=\underline{0}, \underline{1}, \\
\frac{1}{2}, & \text { if } \lambda=\mu_{1}, \\
\frac{1}{3}, & \text { if } \lambda=\mu_{2}, \\
0, & \text { otherwise, }
\end{array} \quad \eta(\lambda)= \begin{cases}1, & \text { if } \lambda=\underline{0}, \underline{1}, \\
\frac{1}{3}, & \text { if } \lambda=\mu_{1}, \mu_{2}, \\
\frac{1}{2}, & \text { if } \lambda=\mu_{3}, \\
0, & \text { otherwise, }\end{cases}\right.
$$

are fuzzy topologies on $X$. Then,

(1) the identity function $\operatorname{id}_{X}:(X, \tau) \rightarrow(X, \eta)$ is FI but not F-super I because $\mu_{3}$ is $1 / 3$-FSO in $(X, \eta)$ and $\tau\left(f^{-1}\left(\mu_{3}\right)\right)=\tau\left(\mu_{3}\right)=0$;

(2) the identity function $\operatorname{id}_{X}:(X, \tau) \rightarrow(X, \tau)$ is FC but not F-super I function.

DEFINITION 2.4. An FTS $(X, \tau)$ is said to be fuzzy extremally disconnected if and only if $\tau\left(C_{\tau}(\lambda, r)\right) \geq r$ for every $\tau(\lambda) \geq r$ for each $\lambda \in I^{X}$ and $r \in I_{\circ}$.

THEOREM 2.5. For a function $f: X \rightarrow Y$, the following statements are true:

(1) if $X$ is fuzzy extremally disconnected and $f$ is FI, then $f$ is F-super I;

(2) if $Y$ is fuzzy extremally disconnected and $f$ is FCI (resp., FC), then $f$ is F-super I;

(3) if both $X$ and $Y$ are fuzzy extremally disconnected, then the concepts F-super I, FCI, FI, FCC, FSC, and FC are equivalent.

Proof. The proof is obvious.

THEOREM 2.6. Let $\left(X, \tau_{1}\right)$ and $\left(Y, \tau_{2}\right)$ be FTSs. Let $f: X \rightarrow Y$ be a function. The following statements are equivalent:

(1) a map $f$ is F-super I;

(2) for each $r$-FSC $\mu \in I^{Y}, \tau\left(\underline{1}-f^{-1}(\mu)\right) \geq r, r \in I_{\circ}$;

(3) for each $\lambda \in I^{X}$ and $r \in I_{\circ}, f\left(C_{T_{1}}(\lambda, r)\right) \leq \mathrm{SC}_{T_{2}}(f(\lambda), r)$;

(4) for each $\mu \in I^{Y}$ and $r \in I_{\circ}, C_{T_{1}}\left(f^{-1}(\mu), r\right) \leq f^{-1}\left(\mathrm{SC}_{T_{2}}(\mu, r)\right)$;

(5) for each $\mu \in I^{Y}$ and $r \in I_{\circ}, f^{-1}\left(\mathrm{SI}_{\tau_{2}}(\mu, r)\right) \leq I_{\tau_{1}}\left(f^{-1}(\mu), r\right)$.

Proof. $(1) \Leftrightarrow(2)$. It is easily proved from Theorem 1.4 and from $f^{-1}(\underline{1}-\mu)=$ $\underline{1}-f^{-1}(\mu)$.

(2) $\Rightarrow$ (3). Suppose there exist $\lambda \in I^{X}$ and $r \in I_{\text {。 }}$ such that

$$
f\left(C_{\tau_{1}}(\lambda, r)\right) \not \mathrm{SC}_{\tau_{2}}(f(\lambda), r) .
$$


There exist $y \in Y$ and $t \in I_{\circ}$ such that

$$
f\left(C_{\tau_{1}}(\lambda, r)\right)(y)>t>\mathrm{SC}_{\tau_{2}}(f(\lambda), r)(y)
$$

If $f^{-1}(\{y\})=\varnothing$, it is a contradiction because $f\left(C_{\tau_{1}}(\lambda, r)\right)(y)=0$.

If $f^{-1}(\{y\}) \neq \varnothing$, there exists $x \in f^{-1}(\{y\})$ such that

$$
f\left(C_{\tau_{1}}(\lambda, r)\right)(y) \geq C_{T_{1}}(\lambda, r)(x)>t>\mathrm{SC}_{\tau_{2}}(f(\lambda), r)(f(x)) .
$$

Since $\mathrm{SC}_{\mathrm{T}_{2}}(f(\lambda), r)(f(x))<t$, there exists $r$-FSC $\mu \in I^{Y}$ with $f(\lambda) \leq \mu$ such that

$$
\mathrm{SC}_{\tau_{2}}(f(\lambda), r)(f(x)) \leq \mu(f(x))<t
$$

Moreover, $f(\lambda) \leq \mu$ implies $\lambda \leq f^{-1}(\mu)$. From (2), $\tau\left(\underline{1}-f^{-1}(\mu)\right) \geq r$. Thus, $C_{\tau_{1}}(\lambda, r)(x) \leq f^{-1}(\mu)(x)=\mu(f(x))<t$, which is a contradiction to (2.6).

(3) $\Rightarrow$ (4). For all $\mu \in I^{Y}, r \in I_{\circ}$, put $\lambda=f^{-1}(\mu)$. From (3), we have

$$
f\left(C_{\tau_{1}}\left(f^{-1}(\mu), r\right)\right) \leq \mathrm{SC}_{\tau_{2}}\left(f\left(f^{-1}(\mu)\right), r\right) \leq \mathrm{SC}_{\tau_{2}}(\mu, r),
$$

which implies that

$$
C_{T_{1}}\left(f^{-1}(\mu), r\right) \leq f^{-1}\left(f\left(C_{T_{1}}\left(f^{-1}(\mu), r\right)\right)\right) \leq f^{-1}\left(\mathrm{SC}_{\tau_{2}}(\mu, r)\right) .
$$

$(4) \Rightarrow(5)$. It is easily proved from Theorem 1.4(1).

(5) $\Rightarrow(1)$. Let $\mu$ be $r$-FSO set of $Y$. From Theorem 1.6(1), $\mu=\mathrm{SI}_{\tau_{2}}(\mu, r)$. By (5),

$$
f^{-1}(\mu) \leq I_{\tau_{1}}\left(f^{-1}(\mu), r\right) .
$$

On the other hand, by Theorem 1.4(2),

$$
f^{-1}(\mu) \geq I_{\tau_{1}}\left(f^{-1}(\mu), r\right) .
$$

Thus, $f^{-1}(\mu)=I_{\tau_{1}}\left(f^{-1}(\mu), r\right)$, that is, $\tau\left(f^{-1}(\mu)\right) \geq r$.

\section{Properties preserved by F-super I functions}

DEFINITION 3.1. Let $(X, \tau)$ be an FTS and $r \in I_{\circ}$. Then

(1) $X$ is called $r$-fuzzy compact (resp., $r$-fuzzy almost compact and $r$-fuzzy nearly compact) if and only if for each family $\left\{\lambda_{i} \in I^{X}: \tau\left(\lambda_{i}\right) \geq r, i \in \Gamma\right\}$ such that $\bigvee_{i \in \Gamma} \lambda_{i}=\underline{1}$, there exists a finite index set $\Gamma_{\circ} \subset \Gamma$ such that $\bigvee_{i \in \Gamma_{0}} \lambda_{i}=\underline{1}$ (resp., $\bigvee_{i \in \Gamma_{\circ}} C_{\tau}\left(\lambda_{i}, r\right)=\underline{1}$ and $\bigvee_{i \in \Gamma_{0}} I_{\tau}\left(C_{T}\left(\lambda_{i}, r\right), r\right)=\underline{1}$ );

(2) $X$ is called $r$-fuzzy semicompact (resp., $r$-fuzzy $S$-closed) if and only if for each family $\left\{\lambda_{i} \in I^{X}: \lambda_{i} \leq C_{\tau}\left(I_{\tau}\left(\lambda_{i}, r\right), r\right), i \in \Gamma\right\}$ such that $\bigvee_{i \in \Gamma} \lambda_{i}=$ $\underline{1}$, there exists a finite index set $\Gamma_{\circ} \subset \Gamma$ such that $\bigvee_{i \in \Gamma_{0}} \lambda_{i}=\underline{1}$ (resp., $\left.\bigvee_{i \in \Gamma_{\circ}} C_{T}\left(\lambda_{i}, r\right)=\underline{1}\right)$. 
THEOREM 3.2. Every surjective F-super I image of $r$-fuzzy compact space is $r$-fuzzy semicompact, $r \in I_{\circ}$.

Proof. Let $(X, \tau)$ be $r$-fuzzy compact, $r \in I_{\circ}$, and let $f:(X, \tau) \rightarrow(Y, \eta)$ be F-super I surjective function. If $\left\{\lambda_{i} \in I^{Y}: \lambda_{i} \leq C_{\eta}\left(I_{\eta}\left(\lambda_{i}, r\right), r\right), i \in \Gamma\right\}$ with $\bigvee_{i \in \Gamma} \lambda_{i}=\underline{1}$, then $\bigvee_{i \in \Gamma} f^{-1}\left(\lambda_{i}\right)=\underline{1}$. Since $f$ is F-super I, $\tau\left(f^{-1}\left(\lambda_{i}\right)\right) \geq r$. Since $X$ is $r$-fuzzy compact, there exists a finite subset $\Gamma_{\circ} \subset \Gamma$ with $\bigvee_{i \in \Gamma_{\circ}} f^{-1}\left(\lambda_{i}\right)=\underline{1}$. From the surjectivity of $f$, we deduce

$$
\underline{1}=f(\underline{1})=f\left(\bigvee_{i \in \Gamma_{\circ}} f^{-1}\left(\lambda_{i}\right)\right)=\bigvee_{i \in \Gamma_{\circ}} f f^{-1}\left(\lambda_{i}\right)=\bigvee_{i \in \Gamma_{\circ}} \lambda_{i}
$$

So, $Y$ is $r$-fuzzy semicompact.

COROLLARY 3.3. Every surjective F-super I image of $r$-fuzzy compact space is $r$-fuzzy $S$-closed, $r \in I_{\circ}$.

THEOREM 3.4. Every surjective F-super I image of $r$-fuzzy almost compact space is $r$-fuzzy $S$-closed, $r \in I_{\circ}$.

PROOF. The proof is similar to that of Theorem 3.2.

COROLLARY 3.5. $r$-fuzzy semicompactness and $r$-fuzzy $S$-closedness are preserved under an F-super I surjection function, $r \in I$.

Proof. The Corollary is a direct consequence of Theorems 3.2 and 3.4.

THEOREM 3.6. Let $f: X \rightarrow Y$ be FSC and F-super I surjective function. If $X$ is $r$-fuzzy nearly compact, then $Y$ is $r$-fuzzy $S$-closed, $r \in I_{\circ}$.

Proof. Let $(X, \tau)$ be $r$-fuzzy nearly compact, and let $r \in I_{\circ}, f:(X, \tau) \rightarrow$ $(Y, \eta)$ be FSC and F-super I surjective function. If $\left\{\lambda_{i} \in I^{Y}: \lambda_{i} \leq C_{\eta}\left(I_{\eta}\left(\lambda_{i}, r\right), r\right)\right.$, $i \in \Gamma\}$ with $\bigvee_{i \in \Gamma} \lambda_{i}=\underline{1}$, then $\bigvee_{i \in \Gamma} f^{-1}\left(\lambda_{i}\right)=\underline{1}$. Since $f$ is F-super I, $\tau\left(f^{-1}\left(\lambda_{i}\right)\right) \geq$ $r$. Since $X$ is $r$-fuzzy nearly compact, there exists a finite subset $\Gamma_{\circ} \subset \Gamma$ with $\bigvee_{i \in \Gamma_{0}} I_{\tau}\left(C_{\tau}\left(f^{-1}\left(\lambda_{i}\right), r\right), r\right)=\underline{1}$. From the surjectivity of $f$, we deduce

$$
\begin{aligned}
\underline{1} & =f(\underline{1})=f\left(\bigvee_{i \in \Gamma_{\circ}} I_{\tau}\left(C_{\tau}\left(f^{-1}\left(\lambda_{i}\right), r\right), r\right)\right) \\
& =\bigvee_{i \in \Gamma_{\circ}} f\left(I_{\tau}\left(C_{\tau}\left(f^{-1}\left(\lambda_{i}\right), r\right), r\right)\right) \\
& \leq \bigvee_{i \in \Gamma_{\circ}} f\left(f^{-1}\left(C_{\eta}\left(\lambda_{i}, r\right)\right)\right) \quad \text { (since } f \text { is FSC [9]). }
\end{aligned}
$$

Thus $\bigvee_{i \in \Gamma_{\circ}} C_{\eta}\left(\lambda_{i}, r\right)=\underline{1}$. Hence $Y$ is $r$-fuzzy $S$-closed. 


\section{Fuzzy $S^{*}$-closed spaces: characterizations and comparisons}

DefinITION 4.1. Let $(X, \tau)$ be an FTS and $r \in I_{\circ}$. Then $X$ is called $r$-fuzzy $S^{*}$-closed if and only if for each family $\left\{\lambda_{i} \in I^{X}: \lambda_{i} \leq C_{\tau}\left(I_{\tau}\left(\lambda_{i}, r\right), r\right), i \in \Gamma\right\}$ such that $\bigvee_{i \in \Gamma} \lambda_{i}=\underline{1}$, there exists a finite index set $\Gamma_{\circ} \subset \Gamma$ such that

$$
\bigvee_{i \in \Gamma_{\circ}} \mathrm{SC}_{\tau}\left(\lambda_{i}, r\right)=\underline{1}
$$

THEOREM 4.2. For an FTS $(X, \tau), r \in I_{\circ}$, the following statements are equivalent:

(1) $X$ is $r$-fuzzy $S^{*}$-closed;

(2) for every family $\left\{\lambda_{i} \in I^{X}: \lambda_{i}\right.$ is $r$-FSCO, $\left.i \in \Gamma\right\}$ such that $\bigvee_{i \in \Gamma} \lambda_{i}=\underline{1}$, there exists a finite index set $\Gamma_{\circ} \subset \Gamma$ such that $\bigvee_{i \in \Gamma_{\circ}} \lambda_{i}=\underline{1}$;

(3) every family of $r$-FSCO sets having the finite intersection property has nonnull intersection;

(4) for every family $\left\{\lambda_{i} \in I^{X}: \lambda_{i}\right.$ is $r$-FSC, $\left.i \in \Gamma\right\}$ such that $\bigwedge_{i \in \Gamma} \lambda_{i}=\underline{1}$, there exists a finite index set $\Gamma_{\circ} \subset \Gamma$ such that $\bigwedge_{i \in \Gamma_{\circ}} \operatorname{SI}_{\tau}\left(\lambda_{i}, r\right)=\underline{1}$.

Proof. $(1) \Rightarrow(2)$. The proof is obvious.

(2) $\Rightarrow(3)$. Let $\left\{\lambda_{i}\right\}_{i \in \Gamma}$ be a family of $r$-FSCO sets having the finite intersection property. If possible, let $\bigwedge_{i \in \Gamma} \lambda_{i}=\underline{0}$. Then $\bigvee_{i \in \Gamma}\left(\underline{1}-\lambda_{i}\right)=\underline{1}$, where each $\left(\underline{1}-\lambda_{i}\right)$ is $r$-FSCO. By (2), there exists a finite subset $\Gamma_{\circ}$ of $\Gamma$ such that $\bigvee_{i \in \Gamma_{\circ}} \underline{1}-\lambda_{i}=\underline{1}$, that is, $\bigwedge_{i \in \Gamma_{\circ}} \lambda_{i}=\underline{0}$, which is a contradiction.

(3) $\Rightarrow(1)$. Suppose that $\left\{\lambda_{i}: i \in \Gamma\right\}$ is a family of $r$-FSO sets of $X$ with $\bigvee_{i \in \Gamma} \lambda_{i}=$ $\underline{1}$, and it has no finite subfamily $\left\{\lambda_{i_{1}}, \ldots, \lambda_{i_{n}}\right\}$ such that $\bigvee_{j=1}^{n} \mathrm{SC}_{\tau}\left(\lambda_{i_{j}}, r\right)=\underline{1}$. Then $\bigwedge_{i=1}^{n}\left(\underline{1}-\operatorname{SC}_{\tau}\left(\lambda_{i_{j}}, r\right)\right) \neq \underline{0}$. Thus, $\left\{\underline{1}-\operatorname{SC}_{\tau}\left(\lambda_{i}, r\right): i \in \Gamma\right\}$ is a family of $r$ FSCO sets having the finite intersection property. By (3), $\bigwedge_{i \in \Gamma}\left(\underline{1}-\mathrm{SC}_{\tau}\left(\lambda_{i}, r\right)\right) \neq$ $\underline{0}$, and hence, $\bigvee_{i \in \Gamma} \lambda_{i} \neq \underline{1}$, which is a contradiction.

(1) $\Rightarrow(4)$. If $\left\{\lambda_{i}: i \in \Gamma\right\}$ is a family of nonnull $r$-FSC sets in $X, r \in I$ 。 with $\bigwedge_{i \in \Gamma} \lambda_{i}=\underline{0}$, then $\left\{\underline{1}-\lambda_{i}: i \in \Gamma\right\}$ is $r$-FSO sets in $X$ with $\bigvee_{i \in \Gamma} \underline{1}-\lambda_{i}=\underline{1}$. By (1), there is a finite subset $\Gamma_{\circ} \subset \Gamma$ such that

$$
\underline{1}=\bigvee_{i \in \Gamma_{\circ}} \mathrm{SC}_{\tau}\left(\underline{1}-\lambda_{i}, r\right)=\underline{1}-\bigwedge_{i \in \Gamma_{\circ}} \mathrm{SI}_{\tau}\left(\lambda_{i}, r\right)
$$

that is, $\bigwedge_{i \in \Gamma_{0}} \operatorname{SI}_{\tau}\left(\lambda_{i}, r\right)=\underline{0}$.

(4) $\Rightarrow(1)$. For any $\left\{\lambda_{i} \in I^{X}: \lambda_{i}\right.$ is $r$-FSO, $\left.i \in \Gamma\right\}$ such that $\bigvee_{i \in \Gamma} \lambda_{i}=\underline{1},\left\{\underline{1}-\lambda_{i}, i \in\right.$ $\Gamma\}$ is a family of $r$-FSC sets such that $\bigwedge_{i \in \Gamma} \underline{1}-\lambda_{i}=\underline{0}$. We can assume, without loss of generality, that each $\underline{1}-\lambda_{i} \neq \underline{0}$. By (4), there is a finite subset $\Gamma_{\circ} \subset \Gamma$ such that $\bigwedge_{i \in \Gamma_{\circ}} \mathrm{SI}_{T}\left(\underline{1}-\lambda_{i}, r\right)=\underline{0}$, that is, $\bigvee_{i \in \Gamma_{\mathrm{o}}} \mathrm{SC}_{\tau}\left(\lambda_{i}, r\right)=\underline{1}$, which proves the $r$-fuzzy $S^{*}$-closedness of $X$.

THEOREM 4.3. Let $(X, \tau)$ be an FTS and $r \in I_{\circ}$. If $X$ is $r$-fuzzy semicompact, then $X$ is $r$-fuzzy $S^{*}$-closed as well. 
Proof. Since for every $\lambda \in I^{X}$ and $r \in I$ 。 we have $\lambda \leq \operatorname{SC}_{T}(\lambda, r)$, this immediately follows from the definitions.

THEOREM 4.4. Let $(X, \tau)$ be an FTS and $r \in I_{\circ}$. If $X$ is $r$-fuzzy $S^{*}$-closed, then $X$ is $r$-fuzzy $S$-closed as well.

PRoof. Since for every $\lambda \in I^{X}$ and $r \in I$ 。 we have $\mathrm{SC}_{T}(\lambda, r) \leq C_{T}(\lambda, r)$, this immediately follows from the definitions.

That the converse is false is evident from the following counterexample.

COUNTEREXAMPLE 4.5. Let $\mathbb{N}$ denote the set of natural numbers with the fuzzy topology $\tau: I^{\mathbb{N}} \rightarrow I$ defined as

$$
\tau(\lambda)= \begin{cases}1, & \text { if } \lambda=\underline{0}, \underline{1} \\ \frac{1}{3}, & \text { if } \lambda=\mu, v \\ \frac{1}{2}, & \text { if } \lambda=\mu \vee v \\ 0, & \text { otherwise, }\end{cases}
$$

where $\mu(1)=1, \mu(i)=0$ (for $i=2,3,4, \ldots$ ), and $\nu(2)=1, \mu(j)=0$ (for $j=$ $1,3,4, \ldots$ ). Let $\rho_{i}^{1}$ and $\rho_{i}^{2}$ (for $i=3,4,5, \ldots$ ) be the fuzzy sets in $I^{\mathbb{N}}$ given by

$$
\begin{aligned}
& \rho_{i}^{1}(x)= \begin{cases}1, & \text { for } x=1 \text { and } i, \\
0, & \text { otherwise, }\end{cases} \\
& \rho_{i}^{2}(x)= \begin{cases}1, & \text { for } x=2 \text { and } i, \\
0, & \text { otherwise. }\end{cases}
\end{aligned}
$$

Then $U=\left\{\rho_{i}^{1}, \rho_{i}^{2}: i=3,4,5, \ldots\right\}$ are $1 / 3$-FSCO sets with $\bigvee_{\rho \in \mathcal{U}} \rho=\underline{1}$ having no finite subcover. Hence $(\mathbb{N}, \tau)$ is not $1 / 3$-fuzzy $S^{*}$-closed, but it is easily seen that $(\mathbb{N}, \tau)$ is $1 / 3$-fuzzy $S$-closed.

THEOREM 4.6. For any fuzzy extremally disconnected $F T S(X, \tau)$ and $r \in I_{\circ}$, $X$ is $r$-fuzzy $S^{*}$-closed if and only if $X$ is $r$-fuzzy $S$-closed.

\section{ProOF}

NECESSITY. It follows from the proof of Theorem 4.4.

SuFFICIENCY. We are going to prove that if $(X, \tau)$ is any fuzzy extremally disconnected FTS, then $C_{\tau}(\lambda, r)=\mathrm{SC}_{\tau}(\lambda, r)$ for every $r$-FSO set $\lambda$ in $(X, \tau)$ and $r \in I_{\text {o }}$. Then our result follows from Definitions 3.1(2) and 4.1.

We always have $\operatorname{SC}_{\tau}(\lambda, r) \leq C_{\tau}(\lambda, r)$ for every $\lambda \in I^{X}$ and $r \in I_{\circ}$. So, we have to prove that with our hypothesis we have $C_{\tau}(\lambda, r) \leq \mathrm{SC}_{\tau}(\lambda, r)$ for every $\lambda \in I^{X}$ and $r \in I_{\circ}$.

If $\lambda$ is $r$-FSO in $(X, \tau)$, then there exists $v \in I^{X}$ with $\tau(v) \geq r$ such that $\nu \leq \lambda \leq C_{\tau}(\nu, r)$. So, $C_{\tau}(\lambda, r)=C_{\tau}(\nu, r)$, where $\tau(\nu) \geq r$. Because $(X, \tau)$ is 
fuzzy extremally disconnected, we have that

$$
C_{\tau}(\lambda, r)=C_{\tau}(\nu, r)=I_{\tau}\left(C_{\tau}(\nu, r), r\right)=I_{\tau}\left(C_{\tau}(\lambda, r), r\right) .
$$

By Lemma 1.7, we have $C_{T}(\lambda, r)=I_{T}\left(C_{T}(\lambda, r), r\right) \leq \mathrm{SC}_{\tau}(\lambda, r)$.

REMARK 4.7. From Theorems 4.3 and 4.4, we have that $r$-fuzzy semicompactness implies $r$-fuzzy $S$-closedness, $r \in I_{\circ}$.

REMARK 4.8. Obviously, for $r \in I_{\circ}, r$-fuzzy $S$-closed space is $r$-fuzzy almost compact. Hence $r$-fuzzy compact space need not be $r$-fuzzy $S^{*}$-closed. That an $r$-fuzzy $S^{*}$-closed space is not necessarily $r$-fuzzy compact is shown by the following counterexample.

CountereXAmple 4.9. Let $X$ be any nonempty set and let $\tau: I^{X} \rightarrow I$ be defined as

$$
\tau(\lambda)= \begin{cases}1, & \text { if } \lambda=\underline{0}, \underline{1}, \\ \frac{1}{2}, & \text { if } \lambda=\underline{\alpha}, \text { for } \frac{1}{2}<\alpha<1 \\ 0, & \text { otherwise. }\end{cases}
$$

Then $(X, \tau)$ is an FTS which is not $1 / 2$-fuzzy compact. Now for any $\underline{\alpha} \in I^{X}$ with $\tau(\underline{\alpha}) \geq 1 / 2, C_{T}(\underline{\alpha}, 1 / 2)=\underline{1}$ and hence $I_{\tau}\left(C_{T}(\underline{\alpha}, 1 / 2), 1 / 2\right)=\underline{1}$, for all $\alpha \in$ $(1 / 2,1]$. Since, by Lemma $1.7, \mathrm{SC}_{\tau}(\underline{\alpha}, 1 / 2)=I_{\tau}\left(C_{\tau}(\underline{\alpha}, 1 / 2), 1 / 2\right)=\underline{1}$, we have for any $r$-FSO set $\lambda, \mathrm{SC}_{\tau}(\lambda, 1 / 2)=\underline{1}$. Hence $X$ is $r$-fuzzy $S^{*}$-closed.

However, we have the following theorem.

THEOREM 4.10. For $r \in I_{\circ}$, every $r$-fuzzy $S^{*}$-closed space is $r$-fuzzy nearly compact, $r \in I_{\circ}$.

Proof. If $X$ is not $r$-fuzzy nearly compact, then there exists $\left\{\lambda_{i} \in I^{X}, i \in \Gamma\right\}$ with $\tau\left(\lambda_{i}\right) \geq r$ and $\bigvee_{i \in \Gamma} \lambda_{i}=\underline{1}$ such that for any finite subset $\Gamma_{\circ} \subset \Gamma$,

$$
\bigvee_{i \in \Gamma_{\circ}} I_{\tau}\left(C_{\tau}\left(\lambda_{i}, r\right), r\right) \neq \underline{1},
$$

that is,

$$
\bigvee_{i \in \Gamma_{\circ}} \operatorname{SC}_{\tau}\left(\lambda_{i}, r\right) \neq \underline{1}
$$

(by Lemma 1.7). Thus, $X$ is not $r$-fuzzy $S^{*}$-closed.

In order to investigate for the condition under which $r$-fuzzy $S^{*}$-closed space is $r$-fuzzy compact, we set the following definition. 
DEFINITION 4.11. An FTS $(X, \tau)$ is called $r$-fuzzy $S$-regular if and only if for each $r$-FSO set $\mu \in I^{X}, r \in I_{\circ}$,

$$
\mu=\bigvee\left\{\rho \in I^{X} \mid \rho \text { is } r \text {-FSO, } \operatorname{SC}_{T}(\rho, r) \leq \mu\right\}
$$

An FTS $(X, \tau)$ is called fuzzy $S$-regular if and only if it is $r$-fuzzy $S$-regular for each $r \in I_{\text {。 }}$.

THEOREM 4.12. If an FTS $(X, \tau)$ is $r$-fuzzy $S$-regular and $r$-fuzzy $S^{*}$-closed, $r \in I_{\circ}$, then it is $r$-fuzzy compact.

Proof. Let $\left\{\lambda_{i} \in I^{X} \mid \tau\left(\lambda_{i}\right) \geq r, i \in \Gamma\right\}$ be a family such that $\bigvee_{i \in \Gamma} \lambda_{i}=\underline{1}$. Since $(X, \tau)$ is $r$-fuzzy $S$-regular, for each $\tau\left(\lambda_{i}\right) \geq r, \lambda_{i}$ is $r$-FSO,

$$
\lambda_{i}=\bigvee_{i_{k} \in K_{i}}\left\{\lambda_{i_{k}} \mid \lambda_{i_{k}} \text { is } r \text {-FSO, } \mathrm{SC}_{\tau}\left(\lambda_{i_{k}}, r\right) \leq \lambda_{i}\right\}
$$

Hence $\bigvee_{i \in \Gamma}\left(\bigvee_{i_{k} \in K_{i}} \lambda_{i_{k}}\right)=\underline{1}$. Since $(X, \tau)$ is $r$-fuzzy $S^{*}$-closed, there exists a finite index $J \times K_{J}$ such that

$$
\underline{1}=\bigvee_{j \in J}\left(\bigvee_{j_{k} \in K_{J}} \operatorname{SC}_{\tau}\left(\lambda_{j_{k}}, r\right)\right)
$$

For each $j \in J$, since

$$
\bigvee_{j_{k} \in K_{J}} \mathrm{SC}_{T}\left(\lambda_{j_{k}}, r\right) \leq \lambda_{j}
$$

we have $\bigvee_{j \in J} \lambda_{j}=\underline{1}$. Hence $(X, \tau)$ is $r$-fuzzy compact.

It is evident that every FI function is FSC. That the converse is not always true is shown in [9]. Again, it is proved in [9] that $f: X \rightarrow Y$ is FI if and only if $f^{-1}(\mu)$ is $r$-FSC for every $r$-FSC set $\mu$ in $Y$ and $r \in I_{\circ}$. Now we have the following theorem.

THEOREM 4.13. The FI image of $r$-fuzzy $S^{*}$-closed space is $r$-fuzzy $S^{*}$-closed, $r \in I_{\text {。 }}$.

THEOREM 4.14. If $f:(X, \tau) \rightarrow(Y, \eta)$ is FI surjective and $X$ is $r$-fuzzy $S^{*}$ closed, then $Y$ is $r$-fuzzy $S$-closed, $r \in I_{\circ}$.

Proof. If $\left\{\lambda_{i} \in I^{Y}: \lambda_{i}\right.$ is $r$-FSO, $\left.i \in \Gamma\right\}$ is a family such that $\bigvee_{i \in \Gamma} \lambda_{i}=\underline{1}$, then $\bigvee_{i \in \Gamma} f^{-1}\left(\lambda_{i}\right)=\underline{1}$. Since $f$ is FI, then, for each $i \in \Gamma, f^{-1}\left(\lambda_{i}\right)$ is $r$-FSO set of $X$. By $r$-fuzzy $S^{*}$-closedness of $X$, there is a finite subset $\Gamma_{\circ} \subset \Gamma$ such that 
$\bigvee_{i \in \Gamma_{\circ}} \mathrm{SC}_{\tau}\left(f^{-1}\left(\lambda_{i}, r\right)\right)=\underline{1}$. Now,

$$
\begin{aligned}
\underline{1} & =f(\underline{1})=f\left(\bigvee_{i \in \Gamma_{\circ}} \mathrm{SC}_{\tau}\left(f^{-1}\left(\lambda_{i}\right), r\right)\right) \\
& \leq f\left(\bigvee_{i \in \Gamma_{\circ}} C_{T}\left(f^{-1}\left(\lambda_{i}\right), r\right)\right) \\
& \leq \bigvee_{i \in \Gamma_{\circ}} C_{\eta}\left(\lambda_{i}, r\right)
\end{aligned}
$$

which implies that $Y$ is $r$-fuzzy $S$-closed.

THEOREM 4.15. If $f:(X, \tau) \rightarrow(Y, \eta)$ is $C I$ surjective and $X$ is $r$-fuzzy nearly compact, then $Y$ is $r$-fuzzy semicompact, $r \in I_{\circ}$.

Proof. The proof is similar to that of Theorem 4.14.

Definition 4.16. Let $(X, \tau)$ and $(Y, \eta)$ be FTSs. A function $f:(X, \tau) \rightarrow$ $(Y, \eta)$ is called semiweakly continuous if and only if

$$
f^{-1}(\lambda) \leq \mathrm{SI}_{\tau}\left(f^{-1}\left(\mathrm{SC}_{\eta}(\lambda, r)\right), r\right)
$$

for each $r$-FSO set $\lambda$ in $(Y, \eta), r \in I_{\circ}$.

THEOREM 4.17. Let $(X, \tau)$ and $(Y, \eta)$ be FTSs and let $f:(X, \tau) \rightarrow(Y, \eta)$ be a semiweakly continuous function. If $X$ is $r$-fuzzy semicompact, then $Y$ is $r$-fuzzy $S^{*}$-closed, $r \in I_{\circ}$.

Proof. If $\left\{\lambda_{i} \in I^{Y}: \lambda_{i}\right.$ is $r$-FSO, $\left.i \in \Gamma\right\}$ is a family such that $\bigvee_{i \in \Gamma} \lambda_{i}=\underline{1}$. From the semiweak continuity of $f$, we have $f^{-1}\left(\lambda_{i}\right) \leq \operatorname{SI}_{\tau}\left(f^{-1}\left(\operatorname{SC}_{\eta}\left(\lambda_{i}, r\right)\right), r\right)$. So, $\mathrm{SI}_{\tau}\left(f^{-1}\left(\mathrm{SC}_{\eta}\left(\lambda_{i}, r\right)\right), r\right)$ is a family of $r$-FSO sets in $(X, \tau)$ with

$$
\bigvee_{i \in \Gamma} \mathrm{SI}_{\tau}\left(f^{-1}\left(\mathrm{SC}_{\eta}\left(\lambda_{i}, r\right)\right), r\right)=\underline{1}
$$

By the semicompactness of $X$, there exists a finite subset $\Gamma_{\circ} \subset \Gamma$ such that $\bigvee_{i \in \Gamma_{\circ}} \operatorname{SI}_{\tau}\left(f^{-1}\left(\operatorname{SC}_{\eta}\left(\lambda_{i}, r\right)\right), r\right)=\underline{1}$. So,

$$
\begin{aligned}
\underline{1} & =f(\underline{1})=f\left(\bigvee_{i \in \Gamma_{\circ}} \mathrm{SI}_{\tau}\left(f^{-1}\left(\mathrm{SC}_{\eta}\left(\lambda_{i}\right), r\right), r\right)\right) \\
& \leq \bigvee_{i \in \Gamma_{\circ}} f f^{-1}\left(\mathrm{SC}_{\eta}\left(\lambda_{i}\right), r\right) \\
& \leq \bigvee_{i \in \Gamma_{\circ}} \mathrm{SC}_{\eta}\left(\lambda_{i}, r\right) .
\end{aligned}
$$

Hence, $\bigvee_{i \in \Gamma_{\circ}} \operatorname{SC}_{\eta}\left(\lambda_{i}, r\right)=\underline{1}$ and $Y$ is $r$-fuzzy $S^{*}$-closed. 
ACKNOWLEDGment. The author is very grateful to the referees.

\section{REFERENCES}

[1] C. L. Chang, Fuzzy topological spaces, J. Math. Anal. Appl. 24 (1968), 182-190.

[2] K. C. Chattopadhyay, R. N. Hazra, and S. K. Samanta, Gradation of openness: fuzzy topology, Fuzzy Sets and Systems 49 (1992), no. 2, 237-242.

[3] K. C. Chattopadhyay and S. K. Samanta, Fuzzy topology: fuzzy closure operator, fuzzy compactness and fuzzy connectedness, Fuzzy Sets and Systems 54 (1993), no. 2, 207-212.

[4] U. Höhle, Upper semicontinuous fuzzy sets and applications, J. Math. Anal. Appl. 78 (1980), no. 2, 659-673.

[5] U. Höhle and A. P. Šostak, A general theory of fuzzy topological spaces, Fuzzy Sets and Systems 73 (1995), no. 1, 131-149.

[6] _ Axiomatic foundations of fixed-basis fuzzy topology, Mathematics of Fuzzy Sets, Handb. Fuzzy Sets Ser., vol. 3, Kluwer Academic Publishers, Massachusetts, 1999, pp. 123-272.

[7] T. Kubiak and A. P. Šostak, Lower set-valued fuzzy topologies, Quaestiones Math. 20 (1997), no. 3, 423-429.

[8] A. A. Ramadan, Smooth topological spaces, Fuzzy Sets and Systems 48 (1992), no. 3, 371-375.

[9] A. A. Ramadan, S. E. Abbas, and Y. C. Kim, Fuzzy irresolute mappings in smooth fuzzy topological spaces, J. Fuzzy Math. 9 (2001), no. 4, 865-877.

[10] A. P. Šostak, On a fuzzy topological structure, Rend. Circ. Mat. Palermo (2) Suppl. (1985), no. 11, 89-103.

[11] _ _ On the neighborhood structure of fuzzy topological spaces, Zb. Rad. (1990), no. 4, 7-14.

[12] _ Basic structures of fuzzy topology, J. Math. Sci. 78 (1996), no. 6, 662-701.

[13] D. Zhang, On the relationship between several basic categories in fuzzy topology, Quaestiones Math. 25 (2002), no. 3, 289-301.

S. E. Abbas: Department of Mathematics, Faculty of Science, South Valley University, Sohag 82524, Egypt

E-mail address: sabbas73@yahoo.com 


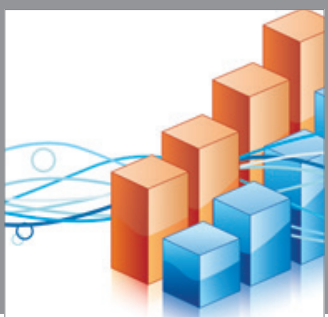

Advances in

Operations Research

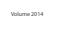

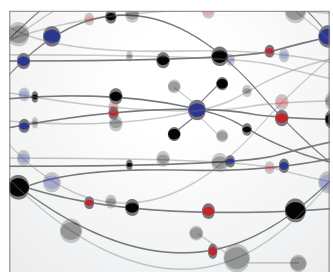

\section{The Scientific} World Journal
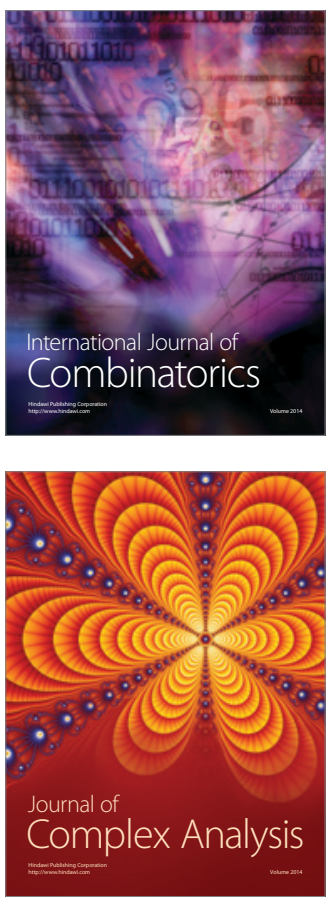

International Journal of

Mathematics and

Mathematical

Sciences
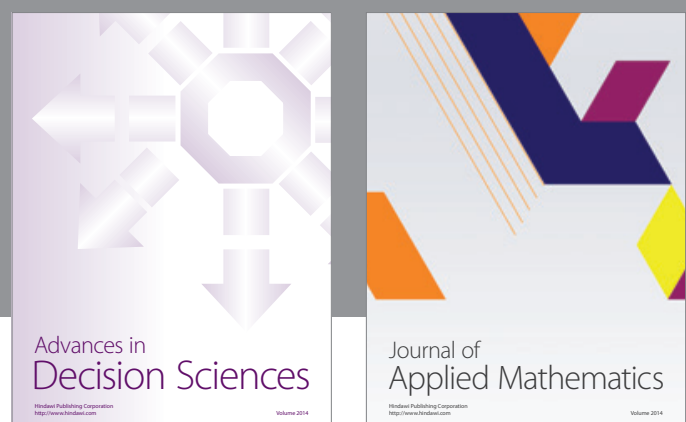

Journal of

Applied Mathematics
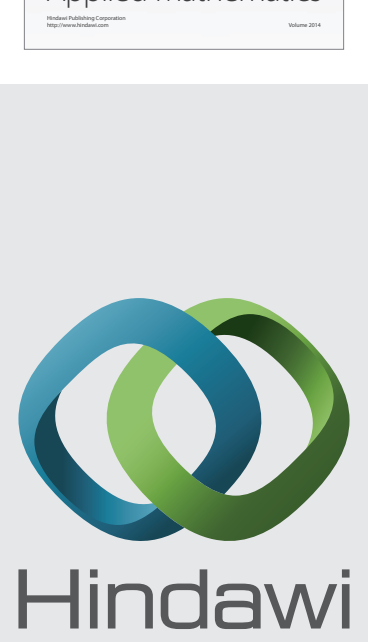

Submit your manuscripts at http://www.hindawi.com
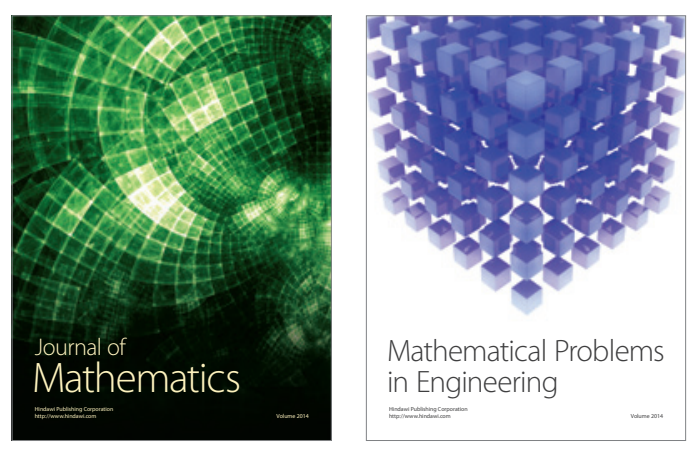

Mathematical Problems in Engineering
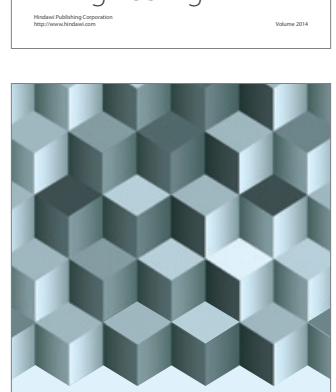

Journal of

Function Spaces
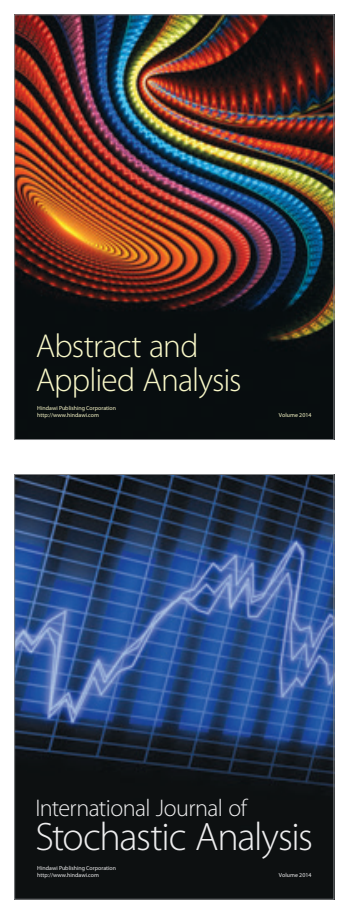

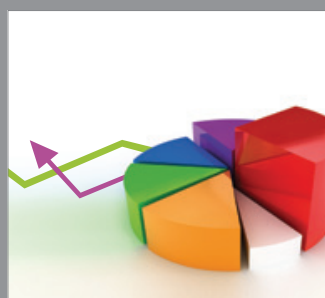

ournal of

Probability and Statistics

Promensencen
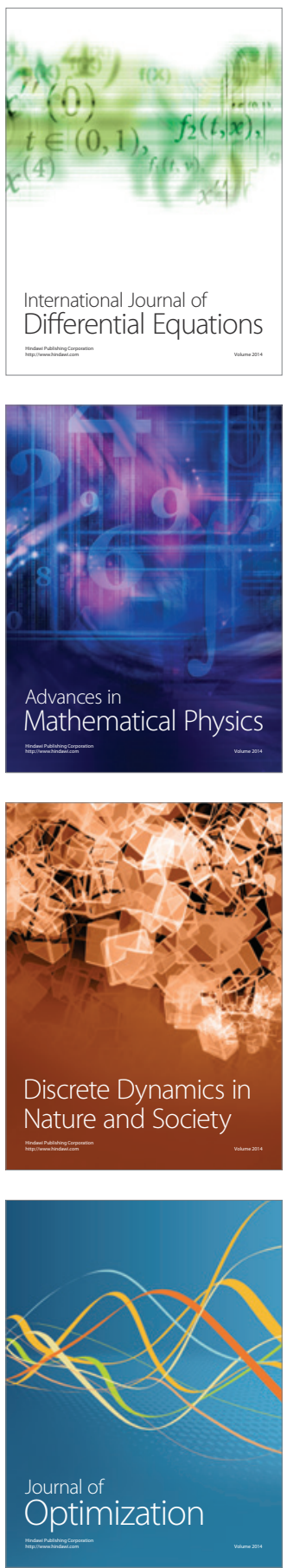